\title{
ERRATUM
}

\section{Conversational responsiveness in specific language impairment}

\section{V. M. BISHOP, JANET CHAN, CATHERINE ADAMS, JOANNE HARTLEY, AND FIONA WEIR}

The following table contained misalignment of the categories and values in the original article published in 2000. Table 3 is reprinted here with the values correctly aligned. The authors regret these errors and any problems they may have caused.

Table 3. Conversational responsiveness

\begin{tabular}{|c|c|c|c|c|c|}
\hline & Mean & $S D$ & Min. & Max. & $N>0$ \\
\hline \multicolumn{6}{|c|}{ No. of solicitations } \\
\hline LA control & 38.22 & 11.04 & 30 & 58 & 0 \\
\hline CA control & 32.00 & 9.27 & 20 & 50 & 0 \\
\hline SLI-T & 36.78 & 8.35 & 27 & 53 & 0 \\
\hline PLI & 30.33 & 8.72 & 13 & 44 & 0 \\
\hline \multicolumn{6}{|l|}{ No response } \\
\hline LA control & 0.033 & 0.037 & 0.000 & 0.100 & 6 \\
\hline CA control & 0.008 & 0.017 & 0.000 & 0.043 & 2 \\
\hline SLI-T & 0.026 & 0.022 & 0.000 & 0.061 & 6 \\
\hline PLI & 0.058 & 0.074 & 0.000 & 0.200 & 5 \\
\hline \multicolumn{6}{|l|}{ Nonverbal } \\
\hline LA control & 0.275 & 0.195 & 0.026 & 0.559 & 9 \\
\hline CA control & 0.120 & 0.093 & 0 & 0.250 & 7 \\
\hline SLI-T & 0.098 & 0.144 & 0 & 0.424 & 5 \\
\hline PLI & 0.047 & 0.076 & 0 & 0.216 & 4 \\
\hline \multicolumn{6}{|l|}{ Prosodic } \\
\hline LA control & 0.014 & 0.023 & 0 & 0.065 & 3 \\
\hline CA control & 0.031 & 0.032 & 0 & 0.080 & 5 \\
\hline SLI-T & 0.052 & 0.060 & 0 & 0.182 & 6 \\
\hline PLI & 0.032 & 0.056 & 0 & 0.162 & 3 \\
\hline \multicolumn{6}{|l|}{ Minimal verbal } \\
\hline LA control & 0.441 & 0.157 & 0.200 & 0.692 & 9 \\
\hline CA control & 0.530 & 0.114 & 0.323 & 0.704 & 9 \\
\hline SLI-T & 0.681 & 0.159 & 0.273 & 0.767 & 9 \\
\hline PLI & 0.659 & 0.124 & 0.459 & 0.870 & 9 \\
\hline \multicolumn{6}{|c|}{ Extended verbal } \\
\hline LA control & 0.238 & 0.088 & 0.088 & 0.367 & 9 \\
\hline CA control & 0.311 & 0.075 & 0.222 & 0.433 & 9 \\
\hline SLI-T & 0.143 & 0.053 & 0.038 & 0.216 & 9 \\
\hline PLI & 0.204 & 0.131 & 0 & 0.400 & 8 \\
\hline
\end{tabular}

Note: LA, language age; CA, chronological age; SLI-T, typical specific language impairment; PLI, pragmatic language impairment. 